\title{
Phase diagrams of classical spin fluids: the influence of an external magnetic field on the liquid-gas transition
}

\author{
W. Fenz, ${ }^{1}$ R. Folk, ${ }^{1}$ I. M. Mryglod, ${ }^{1,2}$ and I. P. Omelyan ${ }^{1,2}$ \\ ${ }^{1}$ Institute for Theoretical Physics, Linz University, A-4040 Linz, Austria \\ ${ }^{2}$ Institute for Condensed Matter Physics, 1 Svientsitskii Street, UA-79011 Lviv, Ukraine
}

(November 3, 2018)

\begin{abstract}
The influence of an external magnetic field on the liquid-gas phase transition in Ising, XY, and Heisenberg spin fluid models is studied using a modified mean field theory and Gibbs ensemble Monte Carlo simulations. It is demonstrated that the theory is able to reproduce quantitatively all characteristic features of the field dependence of the critical temperature $T_{\mathrm{c}}(H)$ for all the three models. These features include a monotonic decrease of $T_{\mathrm{c}}$ with rising $H$ in the case of the Ising fluid as well as a more complicated nonmonotonic behavior for the XY and Heisenberg models. The nonmonotonicity consists in a decrease of $T_{\mathrm{c}}$ with increasing $H$ at weak external fields, an increase of $T_{\mathrm{c}}$ with rising $H$ in the strong field regime, and the existence of a minimum in $T_{\mathrm{c}}(H)$ at intermediate values of $H$. Analytical expressions for $T_{\mathrm{c}}(H)$ in the large field limit are presented as well. The magnetic para-ferro phase transition is also considered in simulations and described within the mean field theory.
\end{abstract}

PACS number(s): 05.70.Fh, 64.60.-i, 64.70.Fx, 75.50.Mm

\section{INTRODUCTION}

The investigation of continuum fluid models with coupled translational and spin degrees of freedom is of current theoretical interest [1-3]. The importance of such models lies in their property to display a rich variety of transitions between solid, liquid, and gas, as well as magnetic ordered and disordered phases, which may occur in real systems. The liquid-gas and magnetic paraferro phase transitions in spin fluids were studied previously by the mean field theory [3-7], the method of integral equations [8-13], and Monte Carlo (MC) simulation techniques $[2,8,11,14-18]$. The theoretical studies dealt mainly with spatially one- $(d=1)$ and three- $(d=3)$ dimensional Ising $(n=1)$ as well as three-dimensional Heisenberg $(n=3)$ fluids (here $n$ denotes the spin dimensionality). In computer experiment, the magnetic transition was investigated for the Heisenberg model $[2,14]$ as well as for three- [16] and two- $(d=2)$ [17] dimensional Ising $(n=1)$ fluids using canonical MC simulations. The combined canonical and Gibbs ensemble MC (GEMC) simulations were performed for a Heisenberg system $(d=3, n=3)$ to determine both the magnetic and liquid-gas transitions $[8,11,15]$.

In most of the previous works, the liquid-gas coexistence was evaluated in the absence $(H=0)$ of an external magnetic field. Only a few papers $[7,11,12]$ were devoted to theoretical study of the fluid behavior at $H \neq 0$. It was found that for systems of hard spheres carrying Ising spins, an external magnetic field decreases the temperature $T_{\mathrm{c}}$ of the gas-liquid critical point [7]. On the other hand, the presence of Heisenberg spins can lead to the inverse effect at strong enough fields [11,12]. As a result, a nonmonotonic behavior of $T_{\mathrm{c}}$ may arise in Heisenberg fluids due to a subtle interplay between the translational and spin degrees of freedom [12]. To our knowledge, no confirmation of the nonmonotonicity in $T_{\mathrm{c}}(H)$ has been given within computer experiment for continuum fluid models of spin systems. In the only work [11] done on GEMC simulations at $H \neq 0$ for a Heisenberg fluid, it has been concluded that the application of an external field increases the gas-liquid critical temperature $T_{\mathrm{c}}$. But this conclusion has been made on the basis of results corresponding to just one finite (sufficiently large) value of $H$.

It is worth mentioning also that, as far as we are aware, no theoretical calculations and computer simulations of $T_{\mathrm{c}}(H)$ have been performed for the planar $(n=2, d=3)$ $\mathrm{XY}$ spin fluid model and no simulations on liquid-gas coexistence in the presence of an external field have been reported for the three-dimensional Ising fluid. Note that we are considering genuine fluid models in which spatial positions of spins are changed continuously (contrary to simplified so-called lattice gas schemes [19-21] where spins are allowed to occupy only positions belonging to sites of a chosen lattice). Moreover, all the works dealt with nonmagnetic repulsion interactions in the form of the simplest hard-sphere potential exclusively. In addition, the magnetic interactions were truncated, as a rule, at some finite interatomic separation, without taking into account long range corrections. The question of how these restrictions impact the behavior of $T_{\mathrm{c}}(H)$ has not been considered as well.

In this paper we present a comprehensive study of the influence of an external magnetic field on the liquid-gas coexistence properties of fluid models with Ising, XY, and Heisenberg spin interactions. The corresponding phase diagrams are calculated in the whole region of varying $H$ with the help of the GEMC simulation technique and a 
modified version of the mean field theory. As is shown, a good agreement between the MC data and theoretical results for $T_{\mathrm{c}}$ can be achieved for all the models and for any value $H$ of the external field, including the limit $H \rightarrow \infty$. At $H=0$, it is demonstrated that the mean field theory predicts a tricritical point independent on the dimension $n$ of the magnetic order parameter. The simulation results for the Ising model agree with this topology, whereas for the XY and Heisenberg fluids a critical end point, beside a gas-liquid critical point in the ferromagnetic phase, is indicated.

\section{MEAN FIELD THEORY}

\section{A. Models}

Let us consider three models of magnetic fluids for spatial dimension $d=3$ with spin interactions of Ising $(n=1)$, planar XY $(n=2)$, and Heisenberg $(n=3)$ types. Within all these models, the total potential energy of the $N$-particle system can be cast in the form

$$
U=\frac{1}{2} \sum_{i \neq j}^{N}\left[\varphi\left(r_{i j}\right)-J\left(r_{i j}\right) \mathbf{s}_{i} \cdot \mathbf{s}_{j}\right]-\mathbf{H} \cdot \sum_{i=1}^{N} \mathbf{s}_{i},
$$

where $\mathbf{r}_{i}=\left(r_{i x}, r_{i y}, r_{i z}\right)$ denotes the spatial coordinate, $\mathbf{s}_{i}$ is the $n$-dimensional spin vector [i.e., $\left(s_{i_{x}}, 0,0\right)$, $\left(s_{i x}, s_{i y}, 0\right)$, or $\left(s_{i x}, s_{i y}, s_{i z}\right)$ for $n=1,2$, or 3 , respectively] of unit length $\left(\left|\mathbf{s}_{i}\right|=1\right)$, and $r_{i j}=\left|\mathbf{r}_{i}-\mathbf{r}_{j}\right|$. For convenience, the homogeneous external magnetic field $\mathbf{H}=(H, 0,0)$ is directed along axis $X$ of the laboratory system of coordinates. The Yukawa function

$$
J(r)=\frac{\epsilon \sigma}{r} \exp \left[\frac{\sigma-r}{\sigma}\right]
$$

is used to describe the internal magnetic interactions, where $\sigma$ and $\epsilon$ relate to the size of particles and coupling constant, respectively. The nonmagnetic interaction $\varphi$ between particles can be modeled by the hard-sphere (HS)

$$
\varphi_{\mathrm{HS}}(r)=\left\{\begin{array}{cc}
\infty, & r<\sigma \\
0, & r \geq \sigma
\end{array}\right.
$$

or soft-core (SC)

$$
\varphi_{\mathrm{SC}}(r)=\left\{\begin{array}{cc}
4 \epsilon\left[\left(\frac{\sigma}{r}\right)^{12}-\left(\frac{\sigma}{r}\right)^{6}\right]+\epsilon, & r<\sqrt[6]{2} \sigma \\
0, & r \geq \sqrt[6]{2} \sigma
\end{array}\right.
$$

repulsion potentials. Note that the attraction between particles is formed exclusively due to ferromagnetic interactions. This corresponds to a so-called "ideal" class of spin fluids, where the attractive part of nonmagnetic interactions is absent (see comments at the end of the paper).

\section{B. Equations of state}

Following the spirit of works [6,22], the Gibbs free energy per particle corresponding to Hamiltonian (1) can be presented as

$$
\mathcal{F}=\mathcal{F}_{\varphi}+\frac{\langle\ln f(\mathbf{s})\rangle}{\beta}+\int_{0}^{1} \mathrm{~d} \alpha \mathcal{F}_{J_{\alpha}}-\mathbf{H} \cdot \mathbf{m}
$$

Here $\mathcal{F}_{\varphi}$ is the free energy of the reference system (in the absence of magnetic interactions and external fields), $f(\mathbf{s})$ relates to the normalized single-particle function describing the distribution of spins in orientational space, $\beta^{-1}=k_{\mathrm{B}} T$ is the temperature with $k_{\mathrm{B}}$ being the Boltzmann's constant, \langle\rangle denotes the statistical averaging, and

$$
\mathbf{m}=\langle\mathbf{s}\rangle=\frac{1}{N}\left\langle\sum_{i=1}^{N} \mathbf{s}_{i}\right\rangle=\int \mathbf{s} f(\mathbf{s}) \mathrm{d} \mathbf{s}
$$

defines the magnetization of the system. The contribution to the free energy caused by spin interactions with the parameterized Yukawa function $J_{\alpha}(r)$ can be written in the form

$$
\mathcal{F}_{J_{\alpha}}=-\frac{\rho}{2} \int g_{\alpha}\left(r, \mathbf{s}_{1}, \mathbf{s}_{2}\right) \frac{\mathrm{d} J_{\alpha}(r)}{\mathrm{d} \alpha} \mathbf{s}_{1} \cdot \mathbf{s}_{2} f\left(\mathbf{s}_{1}\right) f\left(\mathbf{s}_{2}\right) \mathrm{d} \mathbf{r} \mathrm{d} \mathbf{s}_{1} \mathrm{~d} \mathbf{s}_{2},
$$

where $\rho=N / V$ is the number density with $V$ being the volume, and $g_{\alpha}\left(r, \mathbf{s}_{1}, \mathbf{s}_{2}\right)$ introduces the pair distribution function of a system corresponding to $\alpha$-switched on magnetic interactions with $J_{\alpha}=0$ at $\alpha=0$ and $J_{\alpha}=J(r)$ at $\alpha=1$.

Equation (5) formally leads to exact results but requires the knowledge of function $g_{\alpha}\left(r, \mathbf{s}_{1}, \mathbf{s}_{2}\right)$ for each intermediate states $0 \leq \alpha \leq 1$. Since, in general, this function cannot be determined exactly, some approximations are needed to calculate $\mathcal{F}$. Within the mean field (MF) approximation it is assumed that $g_{\alpha}\left(r, \mathbf{s}_{1}, \mathbf{s}_{2}\right)$ does not depend on $\alpha$ and accepts the form of the pair distribution function $g_{\varphi}(r)$ of the reference system. The latter function can further be approximated by its values in the low density regime, $g_{\varphi}(r) \approx \exp [-\beta \varphi(r)]$. Then in view of Eqs. (2) and (6), the integrations in (5) and (7) can be performed explicitly. This results in

$$
\mathcal{F}=\mathcal{F}_{\varphi}+k_{\mathrm{B}} T \int \mathrm{d} \mathbf{s} f(\mathbf{s}) \ln f(\mathbf{s})-\frac{1}{2} a \rho m^{2}-\mathbf{H} \cdot \mathbf{m},
$$

where

$$
a=4 \pi \int_{0}^{\infty} g_{\varphi}(r) J(r) r^{2} \mathrm{dr}=8 \gamma(T) \pi \epsilon \sigma^{3}
$$


is the magnetic interaction strength, and the multiplier

$$
\gamma(T)=\frac{\int_{0}^{\infty} \exp [-\beta \varphi(r)] J(r) r^{2} \mathrm{~d} r}{\int_{\sigma}^{\infty} J(r) r^{2} \mathrm{~d} r}
$$

takes into account the softness of nonmagnetic repulsion potential $\varphi$.

Considering the free energy (8) as a functional of $f(\mathbf{s})$, it can be shown that the minimum of $\mathcal{F}$ is achieved at

$$
f(\mathbf{s})=\frac{\exp (\beta \mathbf{h} \cdot \mathbf{s})}{\int \exp (\beta \mathbf{h} \cdot \mathbf{s}) \mathrm{d} \mathbf{s}},
$$

where

$$
\mathbf{h}=\mathbf{H}+a \rho \mathbf{m}
$$

can be treated as the effective field, consisting of the external term $\mathbf{H}$ and averaged internal contribution $a \rho \mathbf{m}$. Eq. (11) defines, therefore, the equilibrium single-particle distribution function in the MF approximation. Then, taking into account the fact that the vector $\mathbf{m}$ is parallel to $\mathbf{H}$, the right-hand side of Eq. (6) can be integrated in quadratures. The result is

$$
m= \begin{cases}\tanh \left(\frac{H+a \rho m}{k_{\mathrm{B}} T}\right), & n=1, \\ \frac{I_{1}\left(\frac{H+a \rho m}{k_{\mathrm{B}} T}\right)}{I_{0}\left(\frac{H+a \rho m}{k_{\mathrm{B}} T}\right)}, & n=2, \\ \operatorname{coth}\left(\frac{H+a \rho m}{k_{\mathrm{B}} T}\right)-\frac{k_{\mathrm{B}} T}{H+a \rho m}, & n=3,\end{cases}
$$

where $I_{l}(x)=\frac{1}{\pi} \int_{0}^{\pi} \mathrm{e}^{x \cos \psi} \cos (l \psi) \mathrm{d} \psi$ denotes the modified Bessel function of the first kind and order $l$. Relation (13) represents the magnetic equation of state (MES) of the system. Note that the form of this equation depends on the number $n$ of components of the magnetic order parameter $\mathbf{m}$.

The pressure equation of state (PES) can readily be obtained by partially differentiating Eq. (8) with respect to $\rho$, using the thermodynamic relation $P=\rho^{2}(\partial \mathcal{F} / \partial \rho)_{T, H}$ with Eqs. (11) and (12). As a consequence, one finds that the total pressure is the sum of two terms,

$$
P=P_{\varphi}-\frac{1}{2} a \rho^{2} m^{2},
$$

namely, the pressure $P_{\varphi}=\left(\rho^{2} \partial \mathcal{F}_{\varphi} / \partial \rho\right)_{T, H}$ corresponding to the reference system and the part coming from the magnetization. For the HS reference system (3) we use the quasiexact Carnahan-Starling relation [23]

$$
P_{\varphi}(\rho, T)=\rho k_{\mathrm{B}} T\left(1+\eta+\eta^{2}-\eta^{3}\right)(1-\eta)^{-3},
$$

with $\eta=\pi \rho \sigma^{3} / 6=\pi \rho^{*} / 6$ being the packing fraction. In the case of a SC potential (4), the softness of $\varphi$ is taken into account by replacing in Eq. (15) the HS diameter $\sigma$ by its $\mathrm{SC}$ counterpart $\sigma_{\varphi}$. The latter quantity can be determined by requiring the second virial coefficients related to the HS system with the particle's diameter $\sigma_{\varphi}$ and the SC system with the real potential $\varphi$ to be equal between themselves. This leads to

$$
\sigma_{\varphi}(T)=\xi^{1 / 3}(T) \sigma,
$$

where

$$
\xi(T)=\frac{3}{\sigma^{3}} \int_{0}^{\infty}(1-\exp [-\beta \varphi(r)]) r^{2} d r .
$$

Then the SC pressure can be obtained using Eq. (15) with $\eta \equiv \eta_{\varphi}=\pi \rho \sigma_{\varphi}^{3} / 6=\xi(T) \pi \rho^{*} / 6$. This is justified by the fact that the SC potential (4) is close enough to the HS function $(3) \varphi_{\mathrm{SC}}(r)$ increases rapidly to infinity with decreasing $r$ in the range $r<\sigma$, whereas it quickly tends to zero at $r>\sigma)$.

The relations (9), (13) and (14) constitute the main results of the MF theory. In the case of the HS potential (4) (when $g_{\varphi}(r)=1$ for $r \geq \sigma$ and $g_{\varphi}(r)=0$ at $r<\sigma$ and, thus, $\gamma=\xi=1$, see Eqs. (10) and (17)), they coincide completely with those obtained earlier $[5,6]$ (for $n=1$ and 3). Our expressions are more general, since they do not restrict us to the HS convention only, but also are directly applicable for more realistic SC magnetic systems (including the case $n=2$ ).

\section{Phase separations}

Analyzing the MES (13) at $H=0$, it can be shown that nontrivial (nonzero) solutions in the magnetic ordering parameter $m$ take place for temperatures lower than the Curie temperature $T_{\lambda}=a \rho /\left(n k_{\mathrm{B}}\right)$. In the dimensionless representation $\rho^{*}=\rho \sigma^{3}$ and $T^{*}=k_{\mathrm{B}} T / \epsilon$, the magnetic phase transition curve reads $T_{\lambda}^{*}=8 \pi \rho^{*} \gamma\left(T_{\lambda}^{*}\right) / n$. Since, in general, the function $\gamma(T)$ may depend on temperature in a characteristic way, the last equality represents a nonlinear equation which should be solved with respect to $T_{\lambda}^{*}$ at fixed $\rho^{*}$. In the case of SC potential (4), the computations show that the deviations of $\gamma(T)$ from unity do not exceed about $3 \%$ in a wide temperature range of $0.3<T^{*}<6$. For this reason we can put $\gamma(T)=1$ without loss of precision (at least in the range mentioned above). Hence, the Curie temperature is found analytically, $T_{\lambda}^{*}=8 \pi \rho^{*} / n$. It linearly depends on the density and is inversely proportional to the number of spin components. Near the Curie line at $T \lesssim T_{\lambda}$, the MES can be expanded in a series with respect to the deviation $t=\left(T_{\lambda}-T\right) / T_{\lambda}$, yielding

$$
\lim _{t \rightarrow+0} m^{2} \frac{T_{\lambda}}{T_{\lambda}-T}=c,
$$

where $c$ is a constant depending on the spin dimensionality $n$ (see Table I).

In order to get the liquid-gas critical point $T_{\mathrm{c}}$, one has to look where the inverse compressibility goes to zero. The liquid-gas phase transition occurs on the Curie line, 
so that in view of Eqs. (14), (15), and (18) one finds at $T=T_{\lambda}$ that

$$
\beta\left(\frac{\partial P}{\partial \rho}\right)_{T, H}=\frac{\partial}{\partial \eta}\left[\eta \frac{1+\eta+\eta^{2}-\eta^{3}}{(1-\eta)^{3}}\right]-\frac{c n}{2}=0 .
$$

Thus solving Eq. (19) with respect to $\eta$ yields a tricritical point at $\rho_{\mathrm{t}}^{*}=6 \eta_{\mathrm{t}} / \pi$ and $T_{\mathrm{t}}^{*}=8 \pi \rho_{\mathrm{t}}^{*} / n=48 \eta_{\mathrm{t}} / n$. Within the HS version of the MF theory, the solutions $\eta_{\mathrm{t}}$ allow to be presented analytically: $\eta_{\mathrm{t}}=1+[\sqrt{q}-(432 / \sqrt{q}-$ $\left.72-q)^{1 / 2}\right] / 6$ with $q=6(4 p)^{1 / 3}-24-96(2 / p)^{1 / 3}$ and $p=83+3 \sqrt{993}$ for $n=1, \eta_{\mathrm{t}}=1+\sqrt{q}-(1 / \sqrt{q}-1-q)^{1 / 2}$ with $q=\left(p^{1 / 3}-2-17 / p^{1 / 3}\right) / 6$ and $p=82+3 \sqrt{1293}$ for $n=2$, and $\eta_{\mathrm{t}}=1+\left[2 \sqrt{q}-(72 / \sqrt{q}-24-4 q)^{1 / 2}\right] / 6$ with $q=(p / 2)^{1 / 3}-26(2 / p)^{1 / 3}-2$ and $p=245+9 \sqrt{1609}$ for $n=3$. The values for $\rho_{\mathrm{t}}^{*}$ and $T_{\mathrm{t}}^{*}$ with three digits after the decimal point are collected in Table I and marked by the superscript HS. In the case of the $\mathrm{SC}$ version, we should replace (after taking the partial derivative) the packing fraction $\eta$ entering in Eq. (19) by its effective value $\eta_{\varphi}=\xi(T) \pi \rho^{*} / 6$. It can be shown that for the $\mathrm{SC}$ potential defined by Eq. (4), the multiplier $\xi^{1 / 3}(T)$ decreases monotonically from 1.05 to 0.94 with increasing the temperature in the interval $T^{*} \in[0.3,6]$. This behavior has a simple physical meaning, namely, with increasing $T$ the particle can approach to one another more closely due to the increase of their thermal velocities. As a consequence, the effective diameter $\sigma_{\varphi}=\xi^{1 / 3}(T) \sigma$ will decrease. In such a situation (when $\xi \equiv \xi\left(T^{*}\right)$ with $\left.T^{*}=8 \pi \rho^{*} / n\right)$, Eq. (19) transforms into a complicated nonlinear equation in $\rho^{*}$ and must be solved numerically (the integration (17) has been carried out numerically too). The results of these computations are shown in Table I as well and marked by the superscript SC.

TABLE I. Mean field theory results in $d=3$

\begin{tabular}{|c|c|c|c|c|c|c|c|}
\hline Model & $n$ & $c$ & $T_{\lambda}^{*}$ & $\rho_{t}^{* \mathrm{HS}}$ & $T_{t}^{* \mathrm{HS}}$ & $\rho_{t}^{* \mathrm{SC}}$ & $T_{t}^{* \mathrm{SC}}$ \\
\hline Ising & 1 & 3 & $8 \pi \rho^{*}$ & 0.098 & 2.462 & 0.104 & 2.621 \\
\hline $\mathrm{XY}$ & 2 & 2 & $4 \pi \rho^{*}$ & 0.169 & 2.119 & 0.176 & 2.207 \\
\hline Heisenberg & 3 & $\frac{0}{3}$ & $\frac{8 \pi}{3} \rho^{*}$ & 0.224 & 1.876 & 0.229 & 1.920 \\
\hline
\end{tabular}

In the presence of an external field (i.e., when $H \neq 0$ ) there is a liquid-gas phase transition curve ending in a critical point $T_{\mathrm{c}}$. The critical temperature $T_{\mathrm{c}}$ and density $\rho_{\mathrm{c}}$ can be obtained numerically by solving the following system of two equations

$$
\left(\frac{\partial P}{\partial \rho}\right)_{T, H}=0, \quad\left(\frac{\partial^{2} P}{\partial \rho^{2}}\right)_{T, H}=0
$$

where, in view of Eqs. (13) and (14), the pressure $P(\rho, T, m)=P(\rho, T, m(\rho, T, H)) \equiv P(\rho, T, H)$ should be considered as a function of $\rho, T$, and $H$. Therefore, the solutions $T_{\mathrm{c}}(H)$ and $\rho_{\mathrm{c}}(H)$ to system (20) must be found self-consistently with the solution $m \equiv m(\rho, T, H)$ to nonlinear equation (13). The latter equation requires to be handled numerically as well. In the case $n=2$, we have used a representation of the modified Bessel functions (appearing in Eq. (13)) in the form of the infinite series $I_{l}(x)=(x / 2)^{l} \sum_{k=0}^{\infty}(x / 2)^{2 k} /(k !(k+l) !)$ (restricted to a finite but large enough number of terms).

The liquid-gas coexistence curve can be found at $T<$ $T_{\mathrm{c}}$ by applying the Maxwell construction to pressure (14).
Alternatively, we can introduce the chemical potential using the relation $\mu=\mathcal{F}+P / \rho$ and Eqs. (8), (11), and (14). Then one obtains

$$
\mu=\mu_{\varphi}-k_{\mathrm{B}} T \ln \int \exp (\beta \mathbf{h} \cdot \mathbf{s}) \mathrm{d} \mathbf{s},
$$

where

$$
\int \exp (\beta \mathbf{h} \cdot \mathbf{s}) \mathrm{d} \mathbf{s}=\left\{\begin{array}{cc}
2 \cosh \left(\frac{H+a \rho m}{k_{\mathrm{B}} T}\right), & n=1, \\
2 \pi I_{0}\left(\frac{H+a \rho m}{k_{\mathrm{B}} T}\right), & n=2, \\
\sinh \left(\frac{H+a \rho m}{k_{\mathrm{B}} T}\right) & n=3, \\
\frac{H+a \rho m}{k_{\mathrm{B}} T} &
\end{array}\right.
$$

and $\mu_{\varphi}$ is the chemical potential of the reference system which should take its Carnahan-Starling form

$$
\mu_{\varphi}=k_{\mathrm{B}} T\left[\ln \rho+\frac{\eta\left(8-9 \eta+3 \eta^{2}\right)}{(1-\eta)^{3}}\right]
$$


to be self-consistent with Eq. (15). Note that the chemical potential and the pressure factor $Z_{\varphi}(\eta, T)=$ $P_{\varphi}(\rho, T) /\left(\rho k_{\mathrm{B}} T\right)$ are connected by the (exact) relation $\beta \mu_{\varphi}(\eta, T)=\int_{0}^{\eta}\left[Z_{\varphi}\left(\eta^{\prime}, T\right)-1\right] / \eta^{\prime} \mathrm{d} \eta^{\prime}+Z_{\varphi}-1+\ln (\Lambda \rho)$ (the term $k_{\mathrm{B}} T \ln \Lambda$, with $\Lambda$ being the thermal de Broglie wavelength, has been excluded from the right-hand side of Eq. (23), since it depends only on $T$ and is irrelevant for our consideration, see Eq. (24) below). The gas and liquid coexistence densities $\rho_{\mathrm{G}}(T)$ and $\rho_{\mathrm{L}}(T)$ are then determined applying the well-known mechanical and chemical equilibrium conditions

$$
P\left(\rho_{\mathrm{G}}, T\right)=P\left(\rho_{\mathrm{L}}, T\right), \quad \mu\left(\rho_{\mathrm{G}}, T\right)=\mu\left(\rho_{\mathrm{L}}, T\right) .
$$

In the regime of large magnetic fields, we can solve the MES (13) analytically taking into account the smallness of $\zeta=k_{\mathrm{B}} T /(H+a \rho m) \ll 1$. This gives

$$
m_{\zeta \ll 1}= \begin{cases}1-2 \mathrm{e}^{-\frac{2(H+a \rho)}{k_{\mathrm{B}} T}}+\mathcal{O}\left(\mathrm{e}^{-\frac{4}{\zeta^{2}}}\right), & n=1, \\ 1-\frac{1}{2} \frac{k_{\mathrm{B}} T}{H+a \rho}+\mathcal{O}\left(\zeta^{2}\right), & n=2, \\ 1-\frac{k_{\mathrm{B}} T}{H+a \rho}+\mathcal{O}\left(\zeta^{2}\right), & n=3,\end{cases}
$$

where the terms of the second and higher orders for $\mathrm{e}^{-2 / \zeta}$ and $\zeta$ have been omitted, and the inequality $\mathrm{e}^{-2 / \zeta} \ll \zeta$ has been used. Substituting (25) into the PES (14), taking the derivative of $P$ with respect to $\rho$, and solving the resulting equation $(\partial P / \partial \rho)_{T, H}=0$ for $T$, we obtain the critical temperature as a function of $H$. The result is

$$
\frac{T_{\mathrm{c}}(H)-T_{\mathrm{c} \infty}}{T_{\mathrm{c} \infty}}= \begin{cases}4\left(W_{\mathrm{c} \infty}^{\prime}-1\right) \mathrm{e}^{-\frac{2 H}{k_{\mathrm{B}} T_{\mathrm{c} \infty}}}, & n=1, \\ -\frac{k_{\mathrm{B}} T_{\mathrm{c} \infty}}{H}, & n=2, \\ -\frac{2 k_{\mathrm{B}} T_{\mathrm{c} \infty}}{H}, & n=3,\end{cases}
$$

where $T_{\mathrm{c} \infty}=\lim _{H \rightarrow \infty} T_{\mathrm{c}}(H)=a \rho_{\mathrm{c} \infty} /\left(k_{\mathrm{B}} W_{\mathrm{c} \infty}^{\prime}\right)$ and $\rho_{\mathrm{c} \infty}=\lim _{H \rightarrow \infty} \rho_{\mathrm{c}}(H)$ are the critical temperature and density in the infinite magnetic field limit, $W_{\mathrm{c} \infty}^{\prime}=$ $\partial /\left.\partial \eta\left[\eta\left(1+\eta+\eta^{2}-\eta^{3}\right) /(1-\eta)^{3}\right]\right|_{\eta=\eta_{\mathrm{c} \infty}}$ with $\eta_{\mathrm{c} \infty}=$ $\pi \rho_{\mathrm{c} \infty} \sigma^{3} / 6$, and it was assumed that the external field is much larger than both the kinetic energy and the internal magnetic field, i.e., $H \gg k_{\mathrm{B}} T_{\mathrm{c} \infty}$ and $H \gg a \rho_{\mathrm{c} \infty}$. From Eq. (26) we conclude that with increasing the external field, the critical temperature approaches its limiting value from the top, when $n=1$, and from the bottom, when $n=2$ or 3 . Note that the factor $W^{\prime}(\eta)-1>0$ is positive for all physical densities $\eta<1$. Moreover, for the Ising fluid model, the critical temperature $T_{\mathrm{c}}(H)$ tends to $T_{\mathrm{c} \infty}$ exponentially with increasing $H$. This is not the case for XY and Heisenberg fluids, where $T_{\mathrm{c}}(H)$ reaches $T_{\mathrm{c} \infty}$ slower, according to the inverse power law $H^{-1}$.
It is important to remark that in the saturation limit of infinite magnetic field $H \rightarrow \infty$, all the spin fluid models considered reduce to the same (nonmagnetic) fluid with the interparticle potential $\phi(r)=\varphi(r)-J(r)$ consisting of the hard- or soft-core repulsion part as well as the Yukawa-like attraction. The reason is that then the spins align exactly along the field vector, so that the scalar product $\mathbf{s}_{i} \cdot \mathbf{s}_{j}$ will be equal to 1 (see Eq. (1)) for any pairs of particles. The term $\mathbf{H} \cdot \sum_{i} \mathbf{s}_{i}$ will tends to a (infinite) constant and thus can be ignored in Eq. (1) (because we are entitled to accept a new level for counting the energy of the system). The MF theory also leads to identical results for each $n=1,2$, and 3 , when $H \rightarrow \infty$. Indeed, it follows from Eqs. (13) and (25) that the limit $\lim _{H \rightarrow \infty} m=1$ is independent of $n$. The critical temperature and density at such a magnetic saturation, can be found as usually, using the general relation (20) with putting $m=1$ in Eq. (14). This yields $\rho_{\mathrm{c} \infty}^{* \mathrm{HS}} \approx 0.249$ and $T_{\mathrm{c} \infty}^{* \mathrm{HS}} \approx 2.264$ as well as $\rho_{\mathrm{c} \infty}^{* \mathrm{SC}} \approx 0.262$ and $T_{\mathrm{c} \infty}^{* \mathrm{SC}} \approx 2.380$ for the HS and SC versions, respectively. Note also that in the limit $H \rightarrow \infty$, expressions (21) and (22) for the chemical potential can be reduced (by extracting an infinite constant depending only on $H$ ) to the form $\mu=\mu_{\varphi}-a \rho$ that corresponds to a nonmagnetic system with the potential $\phi$.

Simulations show (see the next section) that for the Yukawa-fluid (YF) potential $\phi(r)=\varphi(r)-J(r)$ with the $\mathrm{SC}$ repulsion, the critical liquid-gas temperature is equal to $T_{\mathrm{YF}}^{*} \approx 2.680$. It is somewhat higher (within $12 \%$ ) than the temperature $T_{\mathrm{c} \infty}^{* \mathrm{SC}} \approx 2.380$ obtained within the $\mathrm{SC}$ version of the MF theory. It can be assumed that a significant part of the above temperature discrepancy may come from the approximate form used for the equation of state (see Eq. (15), where $\eta=\xi \pi \rho^{*} / 6$ ) of the SC reference system. Therefore, it becomes quite natural to introduce an adjustable SC (ASC) version of the MF theory, where the second virial parameter $\xi(T)$ of the reference system (Eq. (17)) is replaced by its rescaled analog $\tilde{\xi}(T)=b \xi(T)$. The constant $b$ can then be determined by requiring the critical temperature $T_{\mathrm{YF}}^{*}(b)$ coincides with the exact result. This leads to $b \approx 0.902$ and corresponds to a slight decrease (by a factor of $b^{1 / 3} \approx 0.966$ ) of the effective diameter when calculating the pressure according to Eq. (15) with $\eta=\tilde{\xi}(T) \pi \rho^{*} / 6$.

\section{COMPUTER SIMULATION AND THEORY CALCULATIONS}

\section{A. Simulation procedures}

Two kinds of MC simulations have been carried out to investigate the critical behavior. First, in order to determine the liquid-gas coexistence properties we have applied the Gibbs ensemble MC (GEMC) approach [24] within an advanced biasing scheme [8] for handling spin 
degrees of freedom. The GEMC simulations were performed for two system sizes, namely, with $N=500$ and 1000 particles (or only with $N=500$ or 1000 for some temperatures and nonzero values of $H$ to save computer time). In each case, the particles were distributed over two boxes with volumes which fluctuated under the constraint of fixed total volume. The GEMC configurations were generated in cycles, where one cycle consisted of either (i) $N$ trial displacements and spin reorientations of particles chosen at random; or (ii) one attempted volume rearrangement of the boxes; or (iii) $N_{\text {ex }}$ attempts to exchange the particles between the simulation boxes. The type of each cycle was selected at random and with equal probability among the above three possible steps.

The bias has been used during the spin reorientation and exchange (insertion) steps, so that a new attempted direction for vector $\mathbf{s}_{i}$ was generated with a probability that favors orientations parallel to the local magnetic field. This bias was taken into account when considering the acceptance probabilities corresponding to steps (i) and (iii) as well as when calculating the chemical potential. The analytical expressions for such probabilities have been obtained by modifying the hard-sphere acceptance rules [8] to the case when the soft- (instead of hard-) core nonmagnetic repulsion potential is present. The acceptance ratios for the particle moves and volume changes were adjusted to lie in a range of $30-60 \%$. The value for $N_{\text {ex }}$ was chosen to yield a success rate of particle transfers of $0.1-3 \%$, depending on the temperature. The chemical potential was calculated during the exchange (insertion) step using the generalized Widom's method [25], while the pressure was obtained employing the virial theorem.

Secondly, the Binder crossing technique $[2,26]$ has been utilized to study the magnetic phase transitions (in the absence of an external magnetic field). Here, the usual canonical MC simulations have been performed for system sizes of $N_{1}=250$ and $N_{2}=500$ particles at several particle number densities $\rho$ and temperatures $T$. At each value of $\rho$, the Binder parameter $B=1-M_{4} /\left(3 M_{2}^{2}\right)$ was plotted as a function of $T$ for the two system sizes (this parameter represents a fourth-order cumulant with $M_{l}=\left\langle s^{l}\right\rangle$ and $\left.s=\left|\sum_{i} \mathbf{s}_{i}\right|\right)$. The critical temperature $T_{\lambda}(\rho)$ of the para-ferro phase transition was then obtained from the position of the intersection point of curves $B_{N_{1}}(T)$ and $B_{N_{2}}(T)$, i.e., from the condition $B_{N_{1}}\left(T_{\lambda}\right)=B_{N_{2}}\left(T_{\lambda}\right)$. As in the case of GEMC simulations, the orientational biasing technique [8] was used to improve the convergence of the canonical calculations.

Within both the GEMC and canonical simulations, the Yukawa function was truncated at half of the box edge. In order to reduce the finite-size effects, a long range correction was taken into account (within periodic boundary conditions) by including an additional term to the potential energy of the system. This term was derived [8] by integrating the Yukawa function beyond the cutoff radius and assuming that the binary distribution function is equal to unity in this region. For sufficiently large system sizes (as in our case) such an assumption should lead to virtually exact results. No additional finite size scaling corrections were applied in determining the critical temperatures, assuming that they are small on the scale of phenomena considered. The number of MC cycles used to achieve an equilibrium state varied from $2 \times 10^{4}$ to $5 \times 10^{5}$ depending on the system and external field value. Note that in the GEMC simulations, the pressure and chemical potential should be the same (within statistical noise) at equilibrium in the two (gas and liquid) boxes. After achieving the equilibrium, the investigated quantities were measured by averaging over $2 \times 10^{5}$ to $2.5 \times 10^{6}$ cycles (depending on the model, ensemble, thermodynamic point, and field strength). The statistical uncertainties have been estimated using the block averages method [25].

The critical temperature $T_{\mathrm{c}}$ and density $\rho_{\mathrm{c}}$ have been evaluated at $H \neq 0$ by fitting the discrete set of GEMC data near the criticality to the dependence $\rho_{ \pm}=\rho_{\mathrm{c}}+$ $C_{1}\left(1-T / T_{\mathrm{c}}\right) \pm C_{2}\left(1-T / T_{\mathrm{c}}\right)^{\beta}$ (the constants $C_{1}$ and $C_{2}$ should minimize the deviations between $\rho_{ \pm}$and simulation values). Here the law of rectilinear diameters $\left(\rho_{\mathrm{L}}+\rho_{\mathrm{G}}\right) / 2=\rho_{\mathrm{c}}+C_{1}\left(1-T / T_{\mathrm{c}}\right)$, the power law behavior $\rho_{\mathrm{L}}-\rho_{\mathrm{G}}=2 C_{2}\left(1-T / T_{\mathrm{c}}\right)^{\beta}$, and the critical exponent $\beta=0.32$ have been assumed as in Ref. [27] (where results for a Lennard-Jones fluid were analyzed). Note that for $H \neq 0$ the magnetic para-ferro phase transition disappears and the liquid-gas coexistence curves behave like those of nonmagnetic fluids with the effective attraction potential $J_{\text {eff }}(r)=-\left\langle\mathbf{s}_{1} \cdot \mathbf{s}_{2}\right\rangle_{H} J(r)$. At $H=0$, where both the liquid-gas and magnetic para-ferro transitions exist simultaneously, the application of the above fitting should be performed with care because the rectilinear and power laws will not work properly at temperatures close enough to the temperature $T_{\lambda}$ of the magnetic transition.

\section{B. Results and discussion}

\section{Ising fluid}

Examples of the liquid-gas coexistence obtained in the GEMC simulations for the soft-core Ising fluid at various values, $H^{*}=H / \epsilon=0,0.1,0.5,1,5$, and $\infty$, of the external field are shown in Fig. 1. As can be seen clearly, the critical temperature $T_{\mathrm{c}}$ goes down monotonously with rising the field strength and rapidly tends to its minimal value in the infinite field limit. For instance, already at $H^{*}=5$, the gas and liquid binodal branches are practically indistinguishable from those corresponding to the case $H \rightarrow \infty$. In addition, with increasing $H$ the shape of the coexistence curves becomes wider near a critical point. At $H=0$, all the simulated points belonging to the gas phase lie on a curve which is very close in form to a straight line left to the magnetic para-ferro transition Curie line. The intersection of these lines defines a critical end point which coincides with the liquid-gas critical 
point expected for a tricritical point, i.e., $T_{\mathrm{ce}}=T_{\mathrm{c}}=T_{\mathrm{t}}$. In other words, the Ising fluid exhibits a tricritical behavior. The shape of the Ising binodals has been verified by the multiple-histogram reweighting (MHR) method [28] and agreement with the GEMC results was observed.

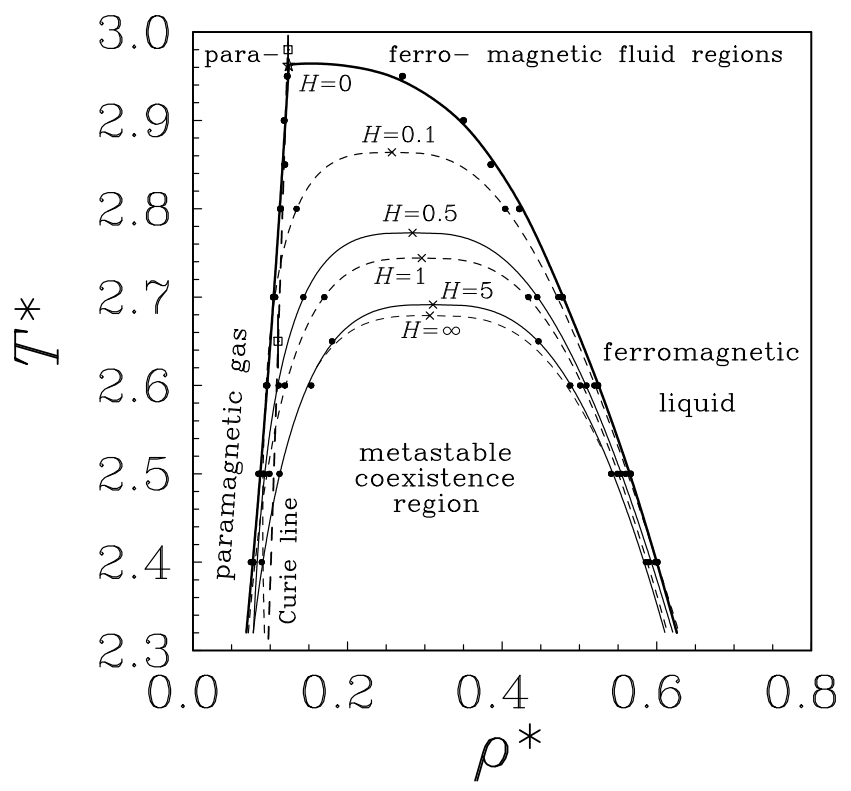

FIG. 1. The liquid-gas coexistence curves obtained from GEMC simulation data (circles) for a soft-core Ising fluid at various values of the external magnetic field $H$. The paraferro phase transition (at $H=0$ ) is plotted by the dashed (Curie) line passing via open squares (obtained in canonical MC simulations). The critical points are shown as crosses, whereas the tricritical point is presented by the star. The values of $H$ are given in the dimensionless form $H^{*}=H / \epsilon$.

In making the above conclusion on the phase diagram topology at $H=0$ we cannot be absolutely sure, since the GEMC simulations (as well as the MHR technique) do not provide us with precise enough data for temperatures which are very close to the critical region. Near this region, the particle fluctuations become too large, so that the two GEMC boxes (which consist of finite numbers of particles) can switch their identity many times during the simulations. This prevents one from obtaining good mean density values of the system in gas and liquid phases at $T \sim T_{\mathrm{c}}$ (although the density distributions over the boxes can still indicate the existence of two phases). On the other hand, having a discrete set of GEMC coexistence points lying relatively far from the criticality region, it is impossible to apply the fitting procedure (see at the end of the preceding subsection) in the vicinity of the expected tricritical point, where $T \sim T_{\lambda}\left(\rho_{\mathrm{c}}\right)=T_{\mathrm{c}}$, because then the liquid and magnetic transitions are coupled. The question concerning the topology of critical points at $H=0$ goes beyond the scope of the present paper and requires additional investigations by more sophisticated simulation techniques (such as finite-size scaling [29], for example).

The primary goal of this work is to study the liquid-gas phase transition in the presence $(H \neq 0)$ of an external field with focusing on the calculations of $T_{\mathrm{c}}$ as a function of $H$. The results of these calculations obtained in the case of the Ising fluid within HS, SC, and ASC versions of the MF theory are shown in Fig. 2 together with the simulation data. Here, a more extended set of external field values has been used. It can be seen that the HSMF and SCMF schemes are able to predict qualitatively the monotonic decrease of the critical temperature $T_{\mathrm{c}}$ with rising $H$. The relative deviations between the simulation data and SCMF predictions for $T_{\mathrm{c}}$ are of order $10-15 \%$, i.e., they are not so large in view of assumptions made within the MF approach. When the ASC version is used, the theoretical and simulation data appear to be practically indistinguishable and the disagreements do not exceed the GEMC statistical noise. At weak external fields $\left(H^{*} \lesssim 0.5\right)$, the function $T_{\mathrm{c}}(H)$ behaves as $T_{\mathrm{c}}(0)-c H^{2 / 5}$ (see Fig. 2, where the latter dependence is plotted at $c=0.258$ for the ASC case). This confirms the MF prediction $\lim _{H \rightarrow 0}\left[T_{\mathrm{c}}(H)-T_{\mathrm{c}}(0)\right] \propto-H^{2 / 5}$, obtained previously in Refs. [7,30]. In the strong field regime $\left(H^{*} \gtrsim 2\right)$, the critical temperature decreases exponentially, according to the analytical formula derived in sect. II.C (see the first line of Eq. (26)).

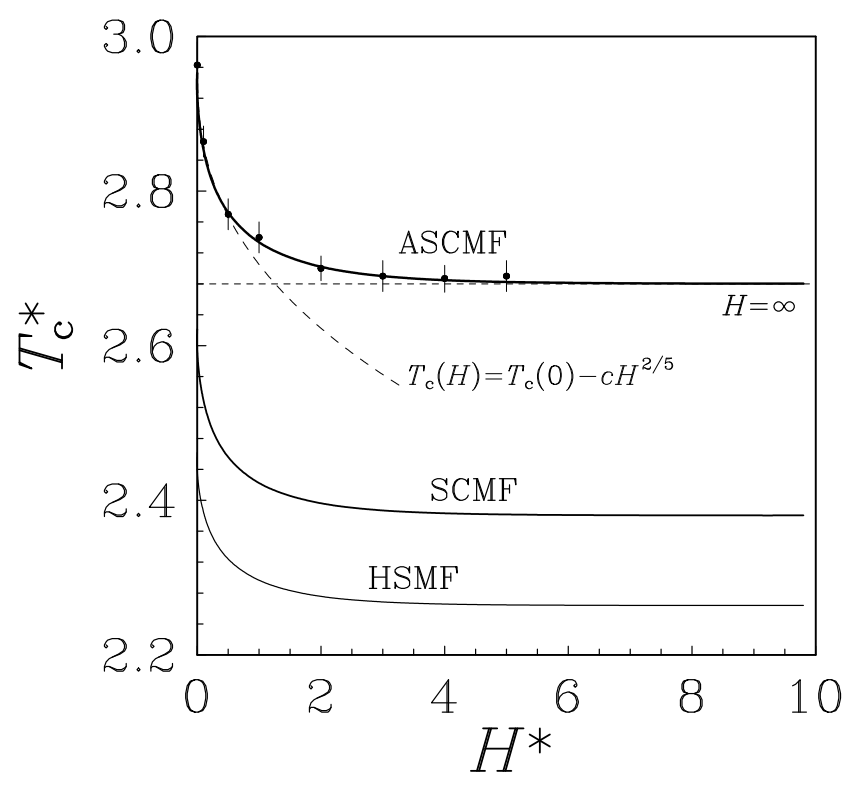

FIG. 2. The critical temperature $T_{\mathrm{c}}$ as a function of the external magnetic field $H$ obtained for the soft-core Ising fluid from GEMC simulations (circles) in comparison with the results of the HS, SC, and ASC versions of the MF theory (solid curves). The critical level corresponding to the infinite value of the external field is plotted by the horizontal dashed line. Vertical bars indicate statistical uncertainties. 
The Ising gas-liquid coexistence curves evaluated within the ASC version of the MF theory as well as the MF para-ferro transition line are presented in Fig. 3. The deviations in the theoretical and simulation binodals (please compare Figs. 1 and 3) are larger than the discrepancy in the case of function $T_{\mathrm{c}}(H)$ (see Fig. 2) and they cannot be reduced to zero even within the ASCMF. For instance, the theory somewhat overestimates the gas phase densities and underestimates the densities in the liquid phase. As a result, the shape of the binodals appears to be narrower with respect to that of the simulation coexistence curves. A more accurate theory should be applied to describe quantitatively the liquid-gas phase diagrams in the whole density, temperature, and magnetic field ranges.

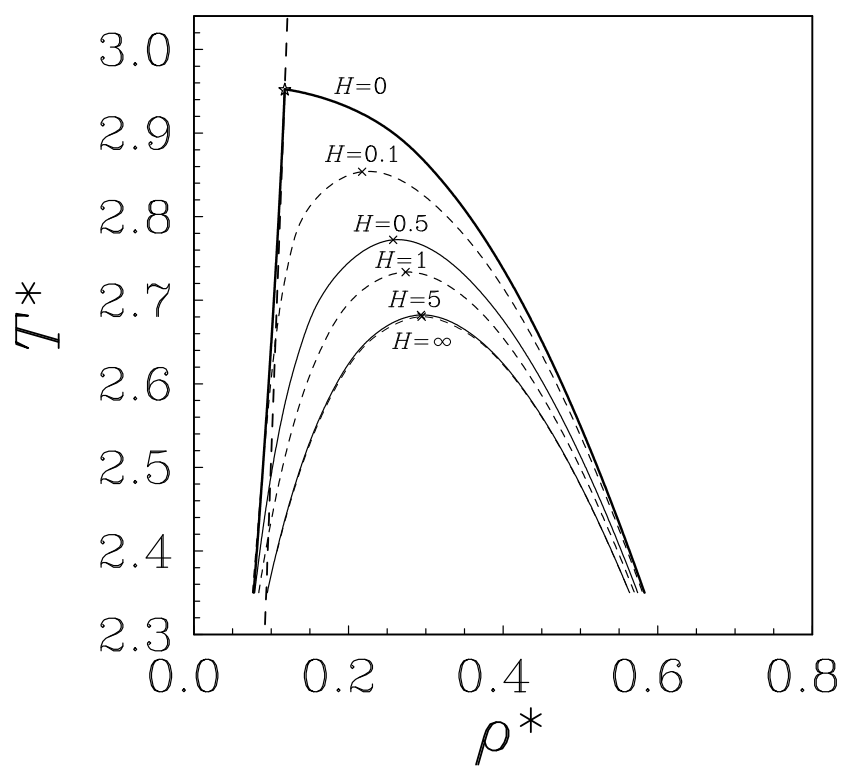

FIG. 3. The gas-liquid coexistence of the Ising fluid obtained within the ASC version of the MF theory. The MF para-ferro transition is plotted by the dashed line. For other notations see the caption to Fig. 1.

\section{XY-spin fluid model}

As was pointed out in the introduction, until now no computer experiment and theoretical investigations on the liquid-gas coexistence have been performed for the planar XY spin fluid model. In this respect it should be mentioned that in order to obtain the coexistence curves within the MF theory, one has to solve numerically Eq. (24). At the same time, the HS, SC, and ASC functions $T_{\mathrm{c}}(H)$ are obtained by finding numerical solutions to Eq. (20) at given values of $H$. Taking into account that the magnetic equation of state (13) must be handled numerically too, the calculation of $\rho_{\mathrm{L}, \mathrm{G}}(T)$ and $T_{\mathrm{c}}(H)$ presents, in fact, a rather complicated technical problem. This is especially true in the case of XY system $(n=2)$ and SC/ASC versions of the MFT, where the integration in Eq. (17) as well as the computation of Bessel's functions are required additionally. Similar difficulties arise in MC simulations of XY fluids when applying the orientational biasing technique [8]. The reason is that then the trial orientation vectors should be generated with distributions, which cannot be presented (contrary to the cases $n=1$ and $n=3$ ) by simple algebraic expressions, and the use of time-consuming Bessel-like functions is required. Nevertheless, developing an efficient algorithm has allowed us to overcome the technical difficulties and calculate the coexistence curves for all the models, including the XY.

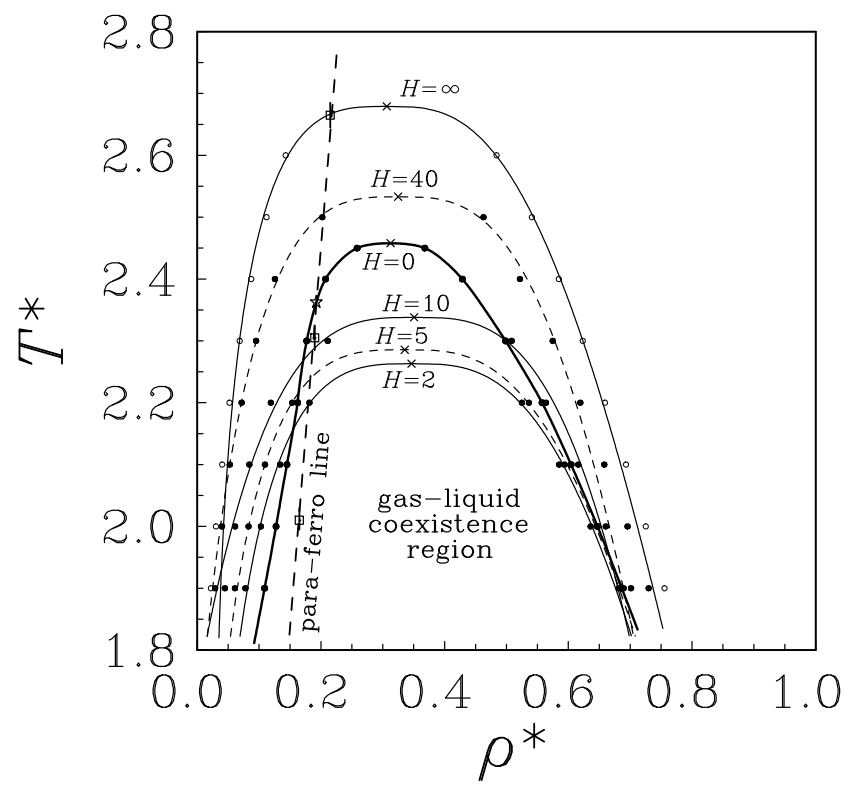

FIG. 4. The gas-liquid (curves) and para-ferro (dashed line) phase coexistences obtained from GE (at different values of an external field $H$ ) and canonical (at $H=0$ ) MC simulations, respectively, for the XY-spin fluid model. The critical end point is shown by the star. Other notations are the same as in Fig. 1.

The XY gas-liquid binodals evaluated in the GEMC simulations are presented in Fig. 4 for the set $H^{*}=0$, $2,5,10,40$, and $\infty$ of external field values. As can be clearly seen, here the topology of phase diagrams differs in several aspects from that of the Ising fluid. First, at $H=0$ the para-ferro magnetic line (which is included in Fig. 4 as well) intersects the gas branch of the binodal at a critical end point, $T_{\mathrm{ce}}$, which does not coincide with the critical point $T_{\mathrm{c}}$, i.e., $\left|T_{\mathrm{c}}-T_{\mathrm{ce}}\right| \neq 0$. So that, contrary to the Ising fluid, where the gas can only be in a paramagnetic state and the liquid be only ferromagnetic, the $\mathrm{XY}$ system exhibits a richer pattern. Here, the gas can 
be either in paramagnetic (when $T<T_{\mathrm{ce}}$ ) or ferromagnetic (when $T_{\text {ce }}<T<T_{\mathrm{c}}$ ) states, and thus the liquid-gas transition can take place with keeping the ferromagnetic ordering. Secondly, with increasing $H$, the critical temperature starts to decrease rapidly reaching its minimal value at $H^{*} \sim 2$ and further begins to increase much slower tending to the infinite-field limit $T_{\mathrm{c} \infty}$. The shape of the binodals near $T_{\mathrm{c}}(H)$ also demonstrates a nonmonotonic behavior, namely, it first becomes wider with rising $H$ and then begins to narrow, but the most sharp shape remains at $H=0$.

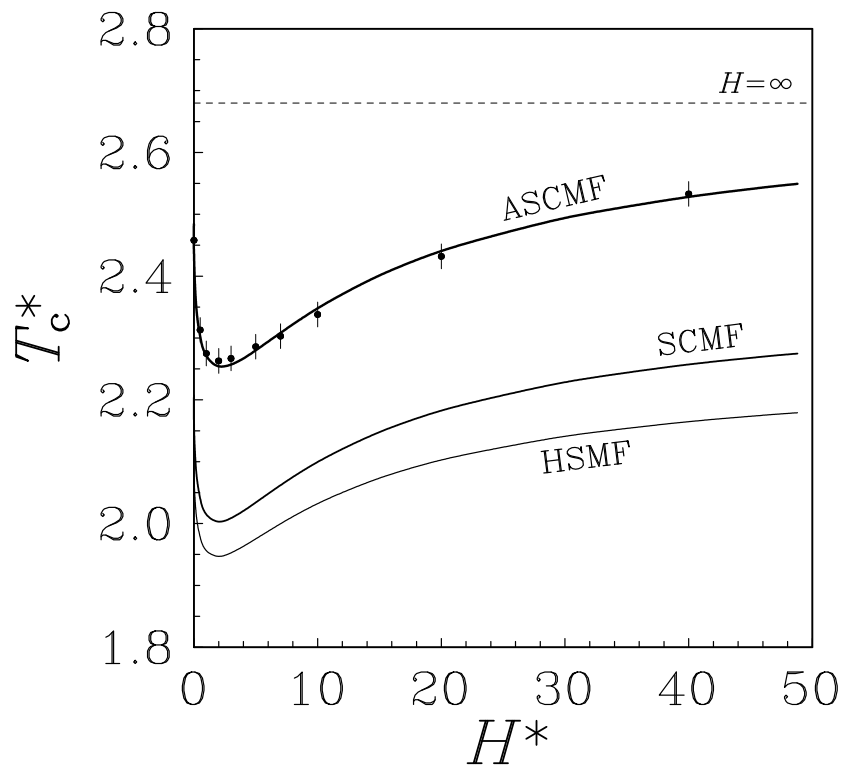

FIG. 5. The critical temperature as a function of the external field obtained for the XY-spin fluid model from GEMC simulations (circles) in comparison with the results of the HS, $\mathrm{SC}$, and ASC versions of the MF theory (solid curves). Other notations are the same as in Fig. 2.

The nonmonotonic dependence of $T_{\mathrm{c}}$ on the strength $H$ of the external field observed from the simulations for the XY fluid is shown in more detail in Fig. 5. The corresponding results of the HS, SC, and ASC MF approaches are included there too. Again we can see that the $\mathrm{HS}$ and $\mathrm{SC}$ versions lead to qualitatively correct results and the deviations from the simulation data do not exceed about $15 \%$. In addition, all the approaches predict a minimum of $T_{\mathrm{c}}(H)$ at nearly the same external field $H^{*} \approx 2$. Moreover, the ASC approach provides us with virtually exact results for $T_{\mathrm{c}}(H)$ at any value of $H$. For instance, the theoretical discrepancy is less than the level of GEMC uncertainties. However, at $H \rightarrow 0$ one has to be careful in application of the MF $H^{2 / 5}$-dependence (see subsection III.B.1) to interpretation of the function $T_{\mathrm{c}}(H)$ build on simulation data. Here, a crossover to an analytic behavior with $\lim _{H \rightarrow 0} \partial T_{\mathrm{c}} / \partial H=0$ is expected because of the lack of a tricritical point $\left(\left|T_{\mathrm{c}}-T_{\text {ce }}\right| \neq 0\right)$. For large fields $\left(H^{*} \gtrsim 50\right)$, the critical temperature tends to the infinite-field value $T_{\mathrm{c} \infty}$ as $T_{\mathrm{c}}(H)-T_{\mathrm{c} \infty} \propto-1 / H$, which is consistent with MF prediction (26) at $n=2$. The saturation regime, when $T_{\mathrm{c}}(H)$ is almost equal to $T_{\mathrm{c} \infty}$, can be achieved here by very strong fields of order $H^{*} \gtrsim 500$ (for the Ising fluid the saturation level is much lower, $H^{*} \gtrsim 5$, see Fig. 2).

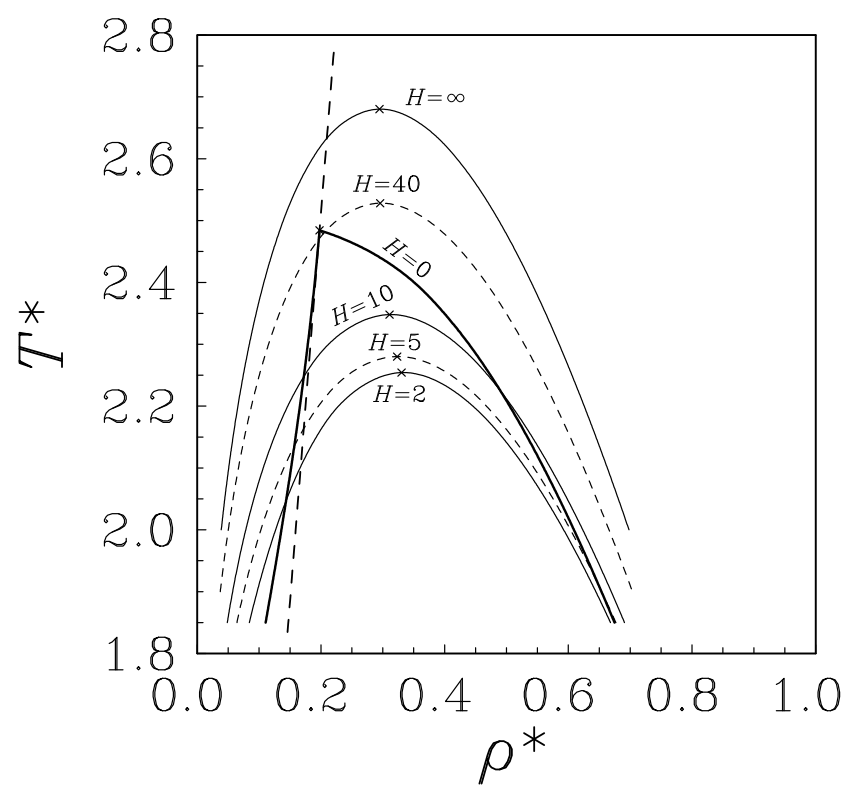

FIG. 6. The gas-liquid and para-ferro coexistences of the $\mathrm{XY}$-spin fluid observed within the ASC version of the MF theory. Notations are similar to those of Fig. 4.

The theoretical liquid-gas coexistence curves and paraferro transition line of the XY fluid are shown in Fig. 6. Comparing these results with the corresponding GEMC data (see Fig. 4) we see that, as in the case of the Ising system, the ASCMF predictions of $\rho_{\mathrm{G}}$ and $\rho_{\mathrm{L}}$ are not as perfect as those of the field dependency of $T_{\mathrm{c}}$. Again, at $H \neq 0$ the shape of the binodals becomes narrower because of the overestimation and underestimation of gas and liquid densities, respectively. For $H=0$, the ASCMF approach does not predict the existence of critical and critical end points found in the simulations, but leads instead to a tricritical behavior.

\section{Heisenberg fluid}

The liquid-gas coexistence curves and the Curie line obtained for the Heisenberg fluid within the simulations and ASCMF theory are shown in Figs. 7 and 8, respectively. As can be seen, the Heisenberg system exhibits a topology of phase diagrams similar to the XY fluid. In particular, according to the simulation results for $H=0$, 
the Curie line ends at a critical end point on the gas side of the binodal, so that $T_{\text {ce }}<T_{\mathrm{c}}$, where $T_{\mathrm{c}}$ should be referred to the liquid-gas critical point located in the ferromagnetic phase (see Fig. 7). It is worth mentioning that some evidence of the lack of a tricritical point in the Heisenberg system has been provided by GEMC simulations earlier [8]. Later on, it was stated [15] that owing to finite size effects it is very difficult to come definitely to one of the two possible scenarios: whether the Curie line ends at a critical end point (as suggested by the simulations, see Fig. 7), or at a tricritical point (as suggested by the MF theory, see Fig. 8). Relatively recently, the existence of a critical end point for the Heisenberg fluid has been observed within the integral equation theory [12]. However, the difference $\left|T_{\mathrm{ce}}-T_{\mathrm{c}}\right|$ reported there was very small and amounted to about $0.1 \%$, which can be comparable with numerical uncertainties. On the other hand, this difference is much smaller than the discrepancy introduced by the mean spherical approximation, used in Ref. [12] for the closure relation to the Ornstein-Zernike equation.

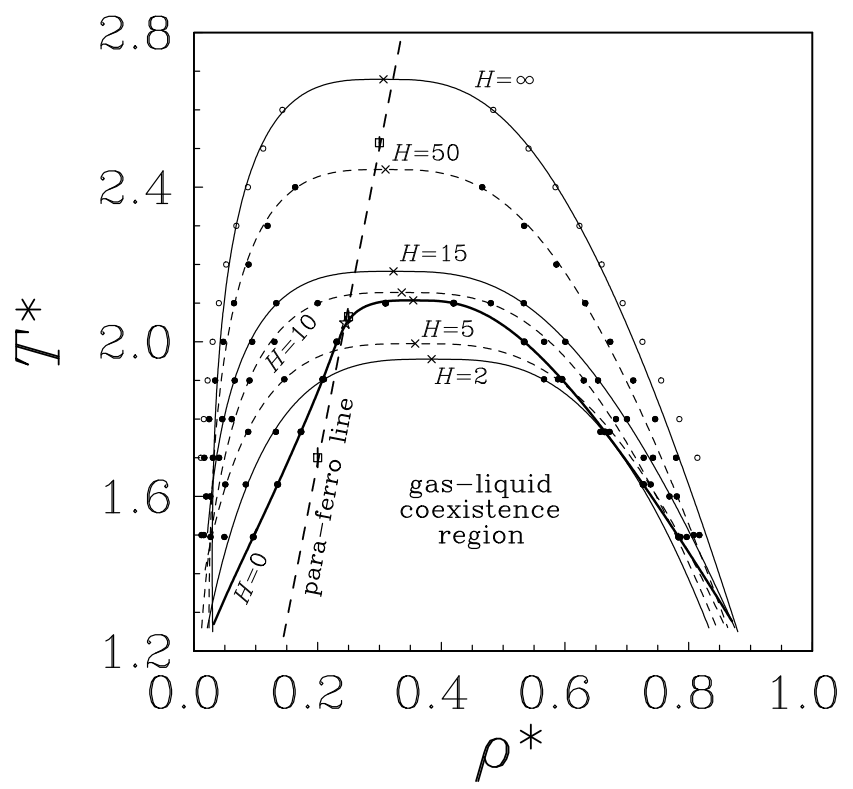

FIG. 7. The gas-liquid (curves) and para-ferro (dashed line) phase coexistence obtained from GE (at different values of an external field $H$ ) and canonical (at $H=0$ ) MC simulations, respectively, for the soft-core Heisenberg fluid. Other notations are the same as in Fig. 4.

In the presence of an external field, the change in shape of the liquid-gas binodal and the change of the critical temperature are nonmonotonic for the Heisenberg fluid like for the XY system. This can be seen from Fig. 7 for the set $H^{*}=0,2,5,10,15,50$, and $\infty$, as well as from Figs. 9 and 10, where the values of $T_{\mathrm{c}}(H)$ are presented for a more complete set of $H$ and in more detail at small
$H$, respectively. Again, the theory is able to qualitatively describe the liquid-gas coexistence properties (see Fig. 8), whereas the function $T_{\mathrm{c}}(H)$ can be calculated within the ASCMF approach quantitatively. The HS and SC versions of the MF theory also reproduce well the critical temperature, but they underestimate $T_{\mathrm{c}}(H)$ to within an order of $10 \%$. The minimum of $T_{\mathrm{c}}(H)$ at $H^{*} \approx 2$ can be predicted by either version of the MFT. It should be pointed out also that the presence of SC repulsion leads to an increase of the gas-liquid critical temperature with respect to that of the HS potential (the SCMF curve lies above the HSMF at all values of $H$, see Figs. 9 and 10). The same conclusion is valid for the Ising and XY models (see Figs. 2 and 5). The asymptotic behavior of $T_{\mathrm{c}}(H)$ at $H^{*} \gg 1$ looks like $T_{\mathrm{c}}(H)-T_{\mathrm{c} \infty} \propto-1 / H$. It is identical in form to that of the XY system, but differs in the value of the coefficient of the proportionality to $1 / H$. Because this coefficient is twice as large at $n=3$ (see Eq. (26)), the saturation regime will begin here at stronger fields $\left(H^{*} \gtrsim 1000\right)$.

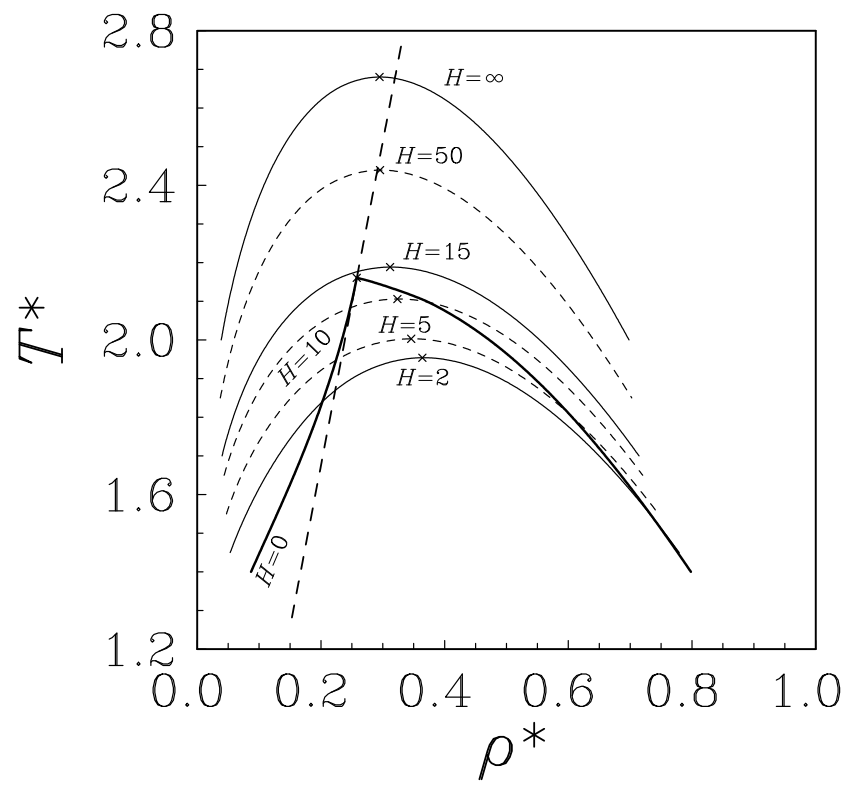

FIG. 8. The gas-liquid and para-ferro coexistences of the Heisenberg fluid obtained within the ASC version of the MF theory. Notations are the same as in Fig. 6.

Note also that in the limit $H \rightarrow 0$, the MF function $T_{\mathrm{c}}(H)$ behaves like $\sim-H^{2 / 5}$ (with $\partial T_{\mathrm{c}} / \partial H \sim$ $\left.-H^{-3 / 5} \rightarrow-\infty\right)$ independently of $n$ (see section IV). However, as in the case of XY fluid, the function $T_{\mathrm{c}}(H)$ corresponding to simulation data for the Heisenberg model (see Figs. 9 and 10) should exhibit an analytic bahavior at $H \rightarrow 0$ with $\lim _{H \rightarrow 0} \partial T_{\mathrm{c}} / \partial H=0$, because of the existence of a critical end point $\left(\left|T_{\mathrm{c}}-T_{\mathrm{ce}}\right| \neq 0\right)$. 


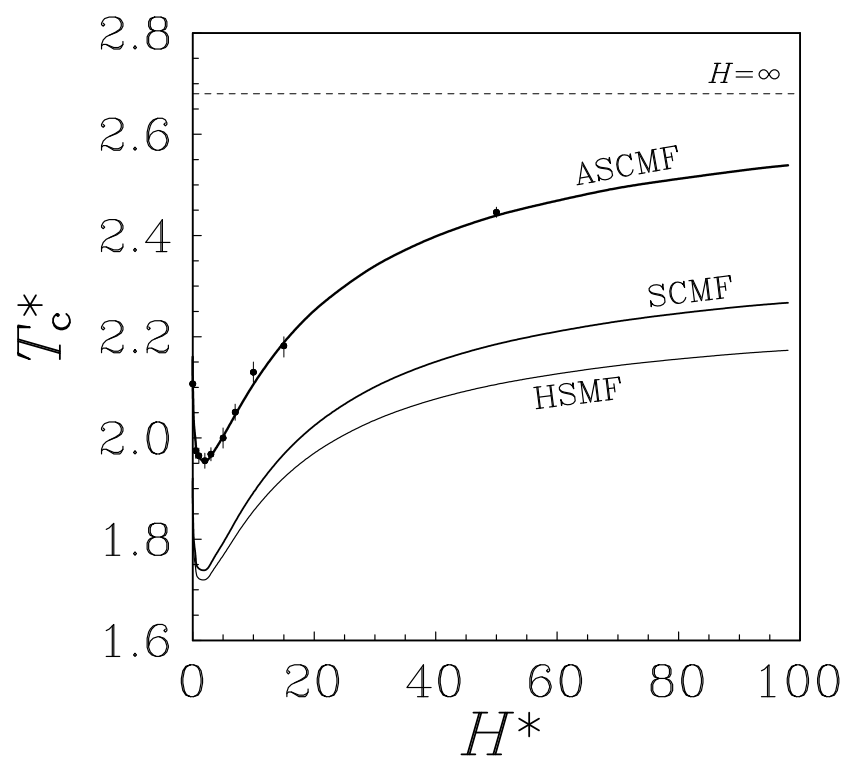

FIG. 9. The critical temperature $T_{\mathrm{c}}$ as a function of the external field $H$ obtained for the Heisenberg fluid from GEMC simulations (circles) in comparison with the results of the HS, $\mathrm{SC}$, and ASC versions of the MF theory (solid curves). Other notations are the same as in Fig. 5.

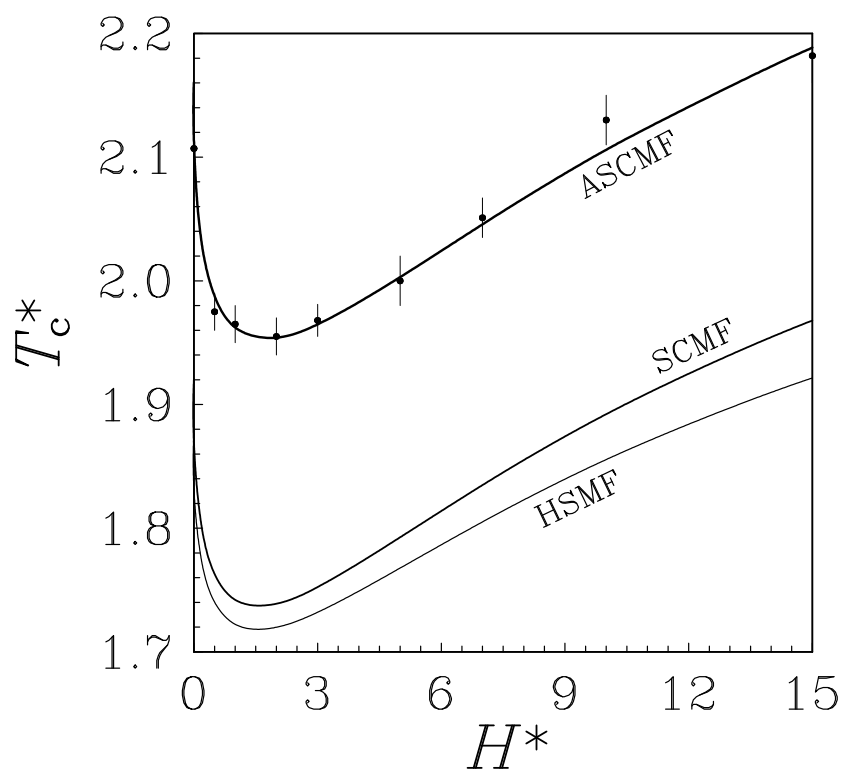

FIG. 10. The same as in Fig. 9, but the behavior of $T_{\mathrm{c}}(H)$ at small values of $H$ is shown in more detail.

For completeness of our consideration we present in detail in Fig. 11 the normalized dependencies $\rho_{\mathrm{c}}(H) / \rho_{\mathrm{c} \infty}$ of the critical density on the external field for the Heisenberg as well as XY, and Ising fluids, obtained within the ASCMF theory in comparison with the GEMC results. The normalization allows to make a quite visible presen- tation of all the functions using only one graph. As can be seen, the simulation points agree well with the ASCMF predictions, although the deviations are somewhat larger than in the case of the field dependency $T_{\mathrm{c}}(H)$ of the critical temperature. The main part of these deviations should be associated with MC statistical noise. For the absolute values of $\rho_{\mathrm{c} \infty}^{*}=\lim _{H \rightarrow \infty} \rho_{\mathrm{c}}^{*}$, related to the ASCMF theory and GEMC simulations, we have obtained 0.295 and 0.307 , respectively. The nonmonotonicity of $\rho_{\mathrm{c}}(H)$ for $n=3$ and 2 is closely connected with the corresponding nonmonotonous behavior of $T_{\mathrm{c}}(H)$. With switching on the external field and its slight increasing, the critical density begins to increase rapidly for all $n=1,2$, and 3. Further, the Heisenberg and XY dependencies $\rho_{\mathrm{c}}(H)$ exhibit a maximum at $H^{*} \approx 2$, i.e., approximately at the same point, where the functions $T_{\mathrm{c}}(H)$ have a minimum (see Figs. 5 and 9). For $n=3$ and 2, after reaching the maximum, the functions $\rho_{\mathrm{c}}(H)$ decrease tending to the same limiting value $\rho_{\mathrm{c} \infty}$. On the other hand, for $n=1$ the function $\rho_{\mathrm{c}}(H)$ continues to increase monotonically to $\rho_{\mathrm{c} \infty}$ on the whole interval of varying $H$ (at the same time, the function $T_{\mathrm{c}}(H)$ monotonically decreases with rising $H$, see Fig. 2).

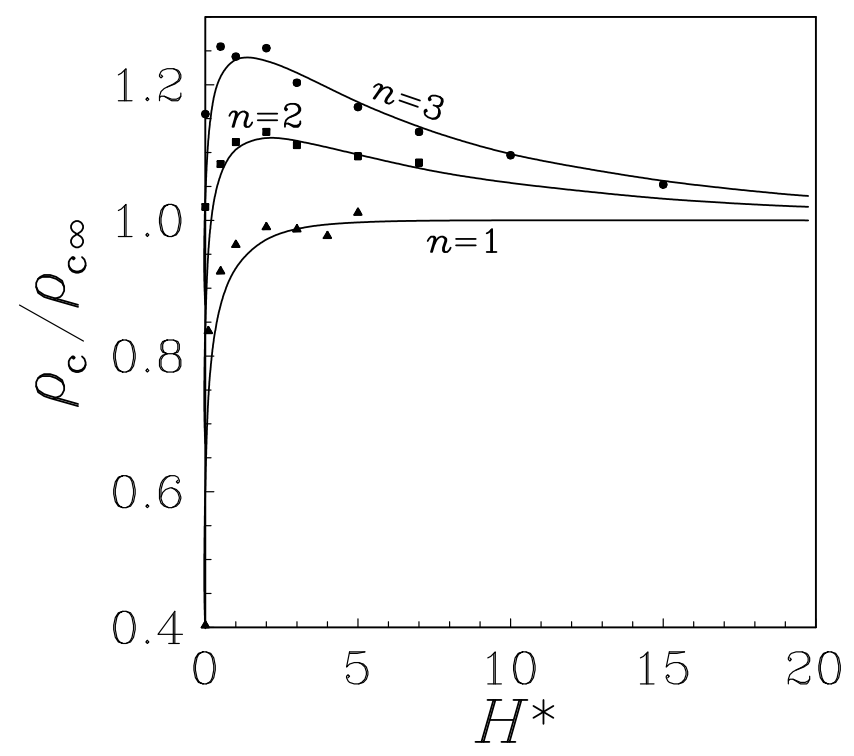

FIG. 11. The normalized critical density $\rho_{\mathrm{c}} / \rho_{\mathrm{c} \infty}$ as a function of the applied external magnetic field $H$, evaluated within the ASCMF theory (curves) for the Ising $(n=1), \mathrm{XY}(n=2)$, and Heisenberg $(n=3)$ spin fluid models. The values obtained from GEMC simulations are presented by triangles, squares, and circles, for $n=1,2$, and 3 , respectively.

Finally, the simulation and theory results on the paraferro coexistence are collected for $n=3,2$, and 1 in Fig. 12. It repeats to some extent the Curie lines already shown in Figs. 1, 3, 4, 6-8, but represents them in a considerably wider temperature interval. Despite the fact 
that only a set of three MC points (for each $n$ ) is available in a restricted density region, it can be stated that the dependence of $T_{\lambda}$ on $\rho$ is almost linear and excellently coincides with the MF straight line $T_{\lambda}^{*}=\frac{8 \pi}{n} \rho^{*}$ (the MC uncertainties are of order of the size of the symbols in Fig. 12).

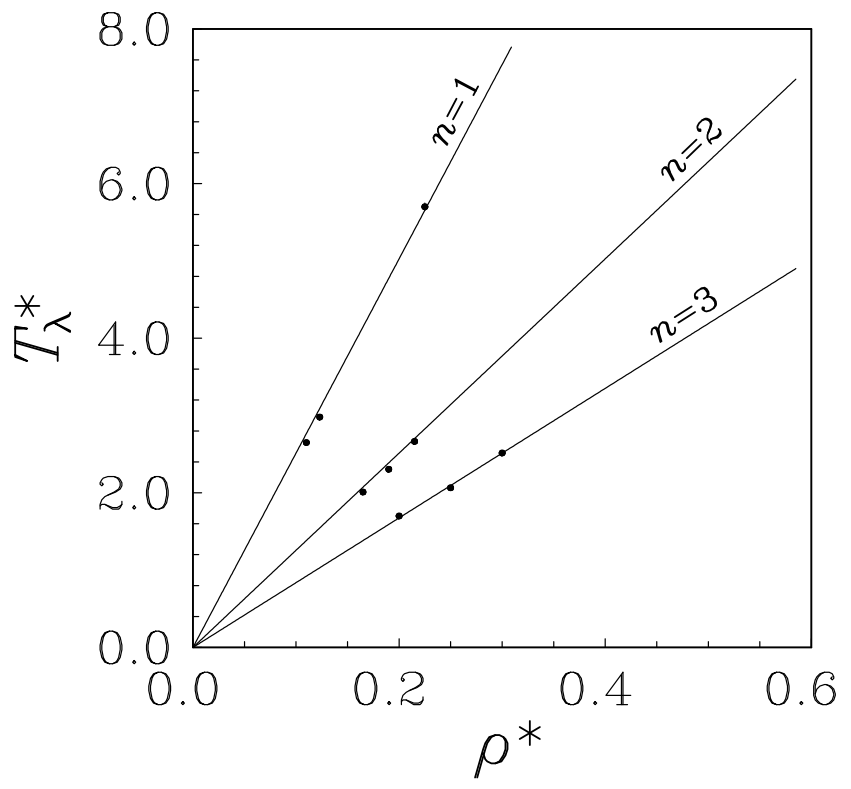

FIG. 12. The para-ferro coexistence lines obtained within the MF theory for the Ising $(n=1), \mathrm{XY}(n=2)$, and Heisenberg $(n=3)$ spin fluid models in comparison with canonical MC simulation data (circles).

\section{CONCLUDING REMARKS}

In the present study we have obtained a complete set of phase diagrams for a class of Ising, XY, and Heisenberg "ideal" spin fluids. The phase diagrams have been calculated applying a new version of the MF theory as well as the Gibbs- and canonical-ensemble MC simulation techniques. The new version takes into account the softness of nonmagnetic interparticle repulsion and corrects the equation of state of the reference system. This has allowed us to describe quantitatively the dependence of the critical temperature $T_{\mathrm{c}}$ of the liquid-gas transition on the strength $H$ of an external magnetic field for all the models considered over the whole region of varying $H$. Let us summarize and discuss the main results found:

(i) It has been established that a common feature inherent in the function $T_{\mathrm{c}}(H)$ is its decrease with turning on and increasing $H$. In the case of the Ising model $(n=1)$, such a behavior remains valid for any further increase of $H$, including the infinite field regime $(H \rightarrow \infty)$. For the XY and Heisenberg models ( $n=2$ and 3$)$, the decrease of $T_{\mathrm{c}}$ with rising $H$ gradually transforms into the inverse dependence, i.e., into an increase of $T_{\mathrm{c}}$ at intermediate and strong fields. In this respect the discrete Ising model (where the spins can accept only two values, +1 or -1 ) exhibits a very specific feature which is not observed for the XY and Heisenberg models with continuous spin distributions.

(ii) From a physical point of view, the field effects just mentioned are caused by the existence of two competing mechanisms. In order to make the explanation more clear, one uses some expressions of the MF theory (which describes already the main features of the field dependency of $T_{\mathrm{c}}$ and $\rho_{\mathrm{c}}$ ). Then the spin fluid can be treated as a simple nonmagnetic system with an effective attraction potential $J_{\text {eff }}(r)=-\left\langle\mathbf{s}_{1} \cdot \mathbf{s}_{2}\right\rangle_{H} J(r)$, where $\left\langle\mathbf{s}_{1} \cdot \mathbf{s}_{2}\right\rangle_{H}=m^{2}$ within the MF approach. This corresponds to the pressure $P=P_{\varphi}-a_{\text {eff }} \rho^{2} / 2$ with $a_{\text {eff }}=a m^{2}$ being the effective attraction strength (see Eq. (14)). Taking into account the dependence of $m$ on $\rho$ (and $H$ ), one obtains that $k_{\mathrm{B}} T_{\mathrm{c}}=\rho a_{\mathrm{eff}}(1+a \rho \chi) / W^{\prime}(\rho)$, where $\chi=\partial m / \partial H$ is the magnetic susceptibility of the system. This result follows from Eq. (15), the condition $(\partial P / \partial \rho)_{T_{\mathrm{c}}}=0$, and the MF relation $\partial m / \partial \rho=a m \chi$ (see Eq. (13)). Differentiating $T_{\mathrm{c}}$ with respect to $H$ yields

$$
\frac{\partial T_{\mathrm{c}}}{\partial H}=\frac{a m \rho}{k_{\mathrm{B}} W^{\prime}}\left[2 \chi(1+a \rho \chi)+a m \rho \frac{\partial \chi}{\partial H}\right],
$$

where the first and second terms on the right-hand side should be associated with the contributions of two different mechanisms. The first mechanism is due to the fact that the external field favors to align the spins along $\mathbf{H}$ and thus increases $m$, i.e., $\chi>0$ for arbitrary values of $H$. For genuine nonmagnetic systems, where $a_{\text {eff }}$ is independent of $\rho$, one obtains the well-known result $k_{\mathrm{B}} T_{\mathrm{c}}=\rho a_{\mathrm{eff}} / W^{\prime}$. This obviously means that the critical temperature is higher for fluids with stronger attractions between particles (e.g., the liquid-gas transition disappears completely for systems with only repulsive interactions, when $a_{\text {eff }}=0$ ). In our case, the effective attractions $a_{\text {eff }}=a m^{2}$ will rise with increasing the magnetic field because of rising $m$, and therefore the critical temperature will increase also (note that the term $2 \chi(1+a \rho \chi)$ remains always positive, see Eq. (27)).

The second mechanism is more subtle and caused by the dependence of $\chi$ on $H$. At strong enough fields $\left(H \gg k_{\mathrm{B}} T\right.$ and $\left.H \gg a \rho\right)$, when the magnetization $m$ is very close to its saturation value, we obtain from Eq. (25) that $\chi=\partial m / \partial H \sim \exp \left[-2 H /\left(k_{\mathrm{B}} T\right)\right]$ for $n=1$ as well as $\chi \sim 1 / H^{2}$ for $n=2$ and 3. Then $\partial \chi /\left.\partial H\right|_{n=1} \sim$ $-\exp \left[-2 H /\left(k_{\mathrm{B}} T\right)\right]<0$ and $\partial \chi /\left.\partial H\right|_{n=2,3} \sim-1 / H^{3}<0$. Thus for $n=2$ and 3 , the influence of the second term in the right-hand side of Eq. (27) on the value $\partial T_{\mathrm{c}} / \partial H$ can be neglected (this term is higher order of smallness in $H$ with respect to the first contribution). In other words, the first mechanism dominates and this indeed is observed for the XY and Heisenberg models, where $T_{\mathrm{c}}$ increases with rising $H$ in the large magnetic field regime. This is, however, not the case for the Ising 
fluid, where the second term (being negative) appears to be the same order in $H$ and even greater in magnitude than the first contribution, owing to the exponential field dependency. Then the critical temperature will decrease with increasing $H$. In particular, for $H \rightarrow \infty$, combining the two terms gives $\lim _{H \rightarrow \infty} k_{\mathrm{B}} \partial T_{\mathrm{c}} / \partial H=$ $-8\left(W_{\mathrm{c} \infty}^{\prime}-1\right) \exp \left[-2 H /\left(k_{\mathrm{B}} T_{\mathrm{c} \infty}\right)\right]<0$.

At weak magnetic fields $H \sim 0$, the second mechanism will prevail for all the models. Following arguments presented in Ref. [7] at $n=1$, it can be shown within the MF approximation that $\partial T_{\mathrm{c}} / \partial H \sim-C(n) H^{-3 / 5}<0$ for any $n=1,2$, and 3 , where $C(n)>0$ is the $n$-dependent coefficient of the proportionality. In order to better understand why the second mechanism suppresses the liquidgas separation, one rewrites the term $a m \rho \partial \chi / \partial H$ in the equivalent form $\rho \partial \chi / \partial \rho-a \rho \chi^{2}$. Then adding it to the first term $2 \chi(1+a \rho \chi)$ results in $2 \chi\left(1+\frac{1}{2} a \rho \chi\right)+\rho \partial \chi / \partial \rho$. Thus, the negative contribution of the second term into the derivative $\partial T_{\mathrm{c}} / \partial H$ directly follows from the fact that the susceptibility of the gaseous phase is larger than that of the coexistent dense liquid phase, i.e., $\partial \chi / \partial \rho<0$. This means that the magnetization of the gaseous phase grows stronger, making the two phases more indistinguishable from one another. This effect is very strong in the limit $H \rightarrow 0$, where the gas branch of the binodal is very close to the Curie line (along which $\chi \rightarrow \infty$ ). At the same time, the liquid branch quickly deviates from this paraferro transition line, leading to especially large negative values of $\partial \chi / \partial \rho$ (with $\lim _{T \rightarrow T_{\mathrm{c}}} \partial \chi / \partial \rho \rightarrow-\infty$ at $H \rightarrow 0$ ).

(iii) We see, therefore, that for the Ising fluid, the second mechanism dominates (owing to the discrete nature of spin reorientations in this case) at all values of $H$. This explains the monotonic decrease of $T_{\mathrm{c}}$ with rising $H$ for $n=1$. With increasing the number $n$ of spin components to 2 and 3 , the ability of the external field to decrease the critical temperature becomes smaller, and the first mechanism begins to prevail at larger values of $H$. This leads to the nonmonotonic behavior of $T_{\mathrm{c}}(H)$ for the XY $(n=2)$ and Heisenberg $(n=3)$ models. The behavior of $\rho_{\mathrm{c}}(H)$ can also be explained by appealing to the interplay between the same two competing processes.

(iv) It has been shown that the MF theory does not predict at $H=0$ the existence of a critical point and critical end point, found in the simulations for $n=2$ and 3 , but leads instead to a tricritical behavior, observed in the simulations for $n=1$. In this context it, first, should be pointed out that we dealt with a specific class of spin fluids, where the attraction between particles is due to ferromagnetic interactions. According to the general classification, this corresponds to an infinite value of the ratio $R=\int g_{\varphi}(r) J(r) \mathrm{d} \mathbf{r} / \int g_{\varphi}(r) \varphi_{\text {attr }}(r) \mathrm{d} \mathbf{r}$ of the integrated strengths of the magnetic interactions and an attractive part of the nonmagnetic interactions, $R=\infty$ (because $\varphi_{\text {attr }}=0$ ). For finite values of $R$, different types of the phase diagram topology can be observed $[5,7]$ within the MF theory (at $R=0$ we come to the usual nonmagnetic fluids). Secondly, for $R=\infty$ and $H=0$, we have concluded that the question on the topology of phase diagrams is very delicate, and requires more accurate theories and more sophisticated simulation techniques. These and related topics will be addressed in further investigations.

\section{ACKNOWLEDGMENT}

Part of this work was supported by the Fonds zur Förderung der wissenschaftlichen Forschung under Project No. P15247. I.M. and I.O. thank the Fundamental Researches State Fund of the Ministry of Education and Science of Ukraine for support under Project No. 02.07/00303.

[1] M. J. P. Nijmeijer and J. J. Weis, in Annual Review of Computational Physics IV, edited by D. Stauffer (World Scientific, Singapore, 1996).

[2] I. M. Mryglod, I. P. Omelyan, and R. Folk, Phys. Rev. Lett. 86, 3156 (2001).

[3] W. Fenz and R. Folk, Phys. Rev. E 67, 021507 (2003).

[4] N. E. Frankel, C. J. Thompson, J. Phys. C: Solid State Phys. 8, 3194 (1975).

[5] P. C. Hemmer and D. Imbro, Phys. Rev. A 16, 380 (1977).

[6] J. M. Tavares, M. M. Telo da Gama, P. I. C. Teixeira, J. J. Weis, and M. J. P. Nijmeijer, Phys. Rev. E 52, 1915 (1995).

[7] F. Schinagl, H. Iro, and R. Folk, Eur. Phys. J. B 8, 113 (1999).

[8] E. Lomba, J.J. Weis, N.G. Almarza, F. Bresme, and G. Stell, Phys. Rev. E 49, 5169 (1994).

[9] T. G. Sokolovska, Physica A 253, 459 (1998).

[10] F. Lado and E. Lomba, Phys. Rev. Lett. 80, 3535 (1998).

[11] F. Lado, E. Lomba, and J. J. Weis, Phys. Rev. E 58, 3478 (1998).

[12] T. G. Sokolovska and R. O. Sokolovskii, Phys. Rev. E 59, R3819 (1999).

[13] E. Lomba, F. Lado, and J. J. Weis, Condens. Matter Phys. 4, 45 (2001).

[14] M. J. P. Nijmeijer and J. J. Weis, Phys. Rev. E 53, 591 (1996).

[15] J. J. Weis, M. J. P. Nijmeijer, J. M. Tavares, and M. M. Telo da Gama, Phys. Rev. E 55, 436 (1997).

[16] M. J. P. Nijmeijer, A. Parola, and L. Reatto, Phys. Rev. E 57, 465 (1998).

[17] A. L. Ferreira and W. Korneta, Phys. Rev. E 57, 3107 (1998).

[18] D. Marx, P. Nielaba, and K. Binder, Phys. Rev. Lett. 67, 3124 (1991).

[19] T. Kawasaki, Prog. Theor. Phys. 58, 1357 (1977).

[20] R. O. Sokolovskii, Phys. Rev. B 61, 36 (2000). 
[21] S. Romano and R. O. Sokolovskii, Phys. Rev. B 61, 11379 (2000).

[22] R. Evans, Adv. Phys. 28, 143 (1979).

[23] N. F. Carnahan and K. E. Starling, J. Chem. Phys. 51, 635 (1969).

[24] A. Z. Panagiotopoulos, N. Quirke, M. Stapleton, and D. J. Tildesley, Mol. Phys. 63, 527 (1988); A. Z. Panagiotopoulos, Molec. Sim. 9, 1 (1992).

[25] D. Frenkel and B. Smit, Understanding Molecular Simulation: from Algorithms to Applications (Academic Press, New York, 1996).
[26] K. Binder, Z. Phys. B 43, 119 (1981); Rep. Prog. Phys. 50, 783 (1987); 60, 487 (1997).

[27] M. J. Kotelyanskii and R. Hentschke, Phys. Rev. E 51, 5116 (1995).

[28] A. M. Ferrenberg and R. H. Swendsen, Phys. Rev. Lett. 61, 2635 (1988); 63, 1195 (1989).

[29] Y. C. Kim and M. E. Fisher, preprint cond-mat/0306331 (2003).

[30] F. Schinagl, R. Folk, and H. Iro, Condens. Matter Phys. 2, 313 (1999). 